\title{
EVALUACIÓN DE LA CONCENTRACIÓN EN UNA RUTA AÉREA BRASILEÑA CON MODELO DEA Y FRONTERA INVERTIDA
}

\section{CONCENTRATION EVALUATION IN A BRASILIAN AIR SHUTTLE WITH DEA MODELS AND INVERTED FRONTIER}

\author{
João Carlos Correia Baptista Soares de Mello ${ }^{1} \quad$ Lidia Angulo Meza $^{2} \quad$ Eliane Gonçalves Gomes $^{3}$ \\ Luiz Biondi Neto ${ }^{4}$ \\ Recibido el 28 de febrero de 2005, aceptado el 28 de julio de 2005 \\ Received: February 28, 2005 Accepted: July 28, 2005
}

\begin{abstract}
RESUMEN
El objetivo de este artículo es promover un primer análisis de las consecuencias para el pasajero del acuerdo de cooperación entre las empresas aéreas Varig y Tam, en una de las principales rutas nacionales. Se utiliza la técnica del Análisis Envolvente de Datos (Data Envelopment Analysis-DEA) con dos fronteras (ópticas del pasajero y del empresario, o frontera tradicional y frontera invertida), para comparar la situación en octubre de 2002 (anterior al acuerdo) y abril de 2003 (posterior). Como los modelos DEA son comparativos, se necesitan incluir en el análisis todas las otras opciones (otras compañías aéreas) de conexiones en la ruta Río de Janeiro-Sao Paulo. Los resultados muestran que dicho acuerdo no beneficia a los pasajeros.
\end{abstract}

Palabras clave: Transporte aéreo, DEA, frontera invertida, eficiencia, compartición.

\section{ABSTRACT}

The main goal of this paper is to provide a preliminary analysis of the code share agreement involving Varig and Tam. The data used is concerned about Rio de Janeiro-Sao Paulo air shuttle, one of the main Brazilian domestic routes. We use Data Envelopment Analysis (DEA) with double frontier approach, to compare the conditions in October 2002 (before the agreement) and April 2003 (after the agreement). As DEA models are comparative ones, we need to extend the analysis to all the other companies flying in the same route. From the results we can say that the agreement did not improve the service provided to passengers.

Keywords: Air transportation, DEA, inverted frontier, efficiency, code share.

\section{INTRODUCCIÓN}

En el inicio del año 2003, con el comienzo del nuevo gobierno brasileño, hubo una inversión de tendencia con relación a la "deregulamentation" o eliminación da reglamentación del sector aéreo (un movimiento que alcanzó el transporte aéreo en EE.UU. durante el gobierno Carter y que se propagó por el mundo). La agenda de esta eliminación había comenzado en 1991, cuando la conexión aérea Río de Janeiro-Sao Paulo (la ruta de mayor tráfico en Brasil, con varios vuelos por hora y por eso conocido como puente aéreo) realizada hasta entonces por un pool de cuatro empresas (Varig, Vasp, Transbrasil y Cruzeiro), pasó a ser realizada de forma independiente por esas empresas y por dos empresas más clasificadas entonces como regionales (Tam, que se convertiría más tarde en una empresa internacional y la empresa regional de Varig, Río Sul).

La situación existente en 1991, además de impedir que los usuarios pudieran escoger, era un factor de concentración perjudicial del transporte aéreo [9]. El inicio de las operaciones por separado fue motivado por la introducción de aviones a propulsión en la ruta y por

\footnotetext{
1 Departamento de Ingeniería de Producción, Universidade Federal Fluminense, jcsmello@producao.uff.br

2 Departamento de Ciencia de los Materiales, Universidad Federal Fluminense, lidia_a_meza@yahoo.com

3 Embrapa, eliane.gomes@cnpm.embrapa.br

4 Departamento de Electrónica y Telecomunicaciones, Universidad del Estado de Rio de Janeiro, lbiondi@uerj.br
} 
una guerra de precios entre el pooly las regionales. Es curioso observar que la lógica empresarial era inexistente en la guerra de precios: Varig actuaba junto con las otras compañías nacionales y luchaba contra su regional Río Sul, aliada a Tam. Tal como se muestra en [13], el objetivo racional de esa guerra era conseguir más pasajeros, sin que existiera una gran preocupación con la generación de lucros. Posteriormente, la guerra de precios se arrastró por todas las empresas que operaban en esa ruta que pasaron a competir entre sí sin ninguna alianza.

Debido a esta situación, hubo un aumento en la competencia, lo que provocó el surgimiento de nuevas opciones de conexión, que pasaron a usar los cuatro aeropuertos disponibles en las dos ciudades. Estos aeropuertos son Santos Dumont (SDU) y Galeón (GIG), en Río de Janeiro y Congonhas (CGH) y Guarulhos (GRU) en Sao Paulo. Santos Dumont y Congonhas tienen una localización central en las respectivas ciudades (principalmente SDU), mientras que Galeón y Guarulhos son más periféricos (en especial GRU).

La eliminación de la reglamentación siguió siendo parte de la agenda del transporte aéreo [7], así se mantuvo la tendencia del aumento de la competencia. El anuncio y el posterior inicio de operaciones conjuntas entre Varig y Tam, en 2003, compartiendo los lugares en sus vuelos (code share) marca una inversión de la agenda.

Por otro lado, la concentración de cerca de $2 / 3$ del PIB brasileño en los Estados de Rio de Janeiro y de Sao Paulo genera un gran tráfico de empresarios y ejecutivos entre las dos ciudades capitales. Este hecho justifica estudios detallados sobre esta ruta. En [15] se presenta un análisis estadístico de las opciones del puente aéreo en abril de 2002.

Este artículo tiene por objetivo evaluar la evolución de las condiciones de oferta en el puente aéreo Río de Janeiro-Sao Paulo en dos momentos: antes y después del anuncio del acuerdo entre Tam y Varig. Se pretende verificar si ese acuerdo, generalmente presentado como solución para los problemas del sector [14], es benéfico para los pasajeros. El estudio usa el Análisis Envolvente de Datos (Data Envelopment Analysis-DEA) con un abordaje de doble óptica ya mostrado en [17] y [8], en el cual las unidades son evaluadas según el punto de vista optimista del pasajero y, en la frontera invertida, según el punto de vista pesimista del pasajero, que también puede ser considerado el punto de vista optimista de la empresa.

\section{ANÁLISIS ENVOLVENTE DE DATOS}

El Análisis Envolvente de Datos (Data Envelopment Analysis-DEA) tiene por objetivo medir la eficiencia de unidades tomadoras de decisión, denominadas DMUs (Decision Making Units), considerando múltiples inputs (entradas, recursos o factores de producción) y múltiples outputs (salidas o productos).

Existen dos modelos DEA llamados clásicos: CCR y BCC. El modelo CCR (también conocido como CRS o Constant Returns to Scale) considera retornos constantes de escala [6]. En su formulación matemática cada DMU $k, k=1, \ldots, n$, es una unidad de producción que utiliza $m$ inputs $x_{i k}, i=1, \ldots, m$, para producir $s$ outputs $y_{j k}, j=1, \ldots, s$.

Este modelo maximiza el cociente entre la combinación lineal de los outputs y la combinación lineal de los inputs, con la restricción de que para cualquier DMU ese cociente no puede ser mayor que 1 .

Mediante algunos artificios matemáticos, ese modelo puede ser linealizado, transformándose en el Problema de Programación Lineal (PPL) mostrado en (1), donde $h_{o}$ es la eficiencia de la DMU o que está siendo analizada; $x_{i o}$ e $y_{j o}$ son los inputs y outputs de la DMU $; v_{i}$ y $u_{j}$ son los pesos calculados por el modelo para inputs y outputs, respectivamente. Este modelo es conocido como modelo CCR.

$$
\begin{aligned}
& \max h_{o}=\sum_{j=1}^{s} u_{j} y_{j o} \\
& \text { sujeto a } \\
& \sum_{i=1}^{m} v_{i} x_{i o}=1 \\
& \sum_{j=1}^{s} u_{j} y_{j k}-\sum_{i=1}^{m} v_{i} x_{i k} \leq 0, \quad k=1, \ldots, n \\
& v_{i}, u_{j} \geq 0 \quad \forall i, j
\end{aligned}
$$

El modelo BCC [4], también llamado de VRS (Variable Returns to Scale), considera situaciones de eficiencia de producción con variación en la escala y no asume proporcionalidad entre inputs youtputs. El modelo BCC no será utilizado en este trabajo.

A pesar de que los modelos DEA tienen la ventaja de hacer posible ordenaciones, según la eficiencia obtenida por el modelo sin depender de las opiniones de los decidores, ellos son extremamente benevolentes con las unidades evaluadas. Éstas pueden ser eficientes al considerar apenas algunas de las variables, a saber, aquellas que les son más favorables. Esa característica 
de benevolencia de los modelos DEA hace que ocurran empates para las unidades $100 \%$ eficientes, lo que ocasiona una baja discriminación entre las DMUs.

Existen varios métodos para evitar el problema de baja discriminación [2]. Este artículo propone utilizar la media aritmética de la eficiencia según la óptica de los pasajeros (lo que caracteriza una conexión eficiente según el punto del vista del pasajero) y de la eficiencia según la óptica de los empresarios (lo que caracteriza una conexión eficiente según el punto de vista del empresario). Estas dos ópticas serán detalladas en la próxima sección.

Cada una de las ópticas mencionadas construye una frontera DEA CCR, mutuamente invertidas. La media aritmética mencionada y mostrada en (2) es igual al método de agregación usado por [11].

Eficiencia $_{\text {final }}=\frac{\text { Eficiencia }_{\text {pasajero }}-\text { Eficiencia }_{\text {empresário }}+100}{2}$

En este modelo se puede considerar que la eficiencia en la óptica del pasajero representa la eficiencia DEA clásica, es decir, se trata de una evaluación optimista. La eficiencia, según la óptica del empresario, se interpreta como una frontera invertida [12] que, según [8], se obtiene con el cambio de inputs por outputs y viceversa y representa una evaluación pesimista.

El uso de las dos fronteras también permite que se evite otro problema de DEA que tiende a ignorar algunas de las variables de la evaluación. Por otro lado, no son necesarias evaluaciones subjetivas de pesos o importancia de criterios de evaluación (inputs y outputs) que es una forma de conseguir el aumento de discriminación y la consideración de todas las variables relevantes.

Para obtener un buen índice de eficiencia, una DMU debe mostrar buen desempeño en las variables que le son favorables y no debe mostrar un mal desempeño en las variables que le son desfavorables.

\section{MODELAMIENTO}

\section{Estructuración}

Para estructurar un problema DEA se necesita definir las DMUs o unidades que serán evaluadas, las variables de evaluación (inputs y outputs) y el modelo DEA que será utilizado (CCR, BCC, entre otros).
Las unidades que están siendo evaluadas son las diferentes opciones que un pasajero tiene para hacer la conexión aérea entre Río de Janeiro y Sao Paulo. Los datos utilizados son de octubre de 2002 y de abril de 2003. La elección de esas fechas refleja los dos momentos de interés en este artículo, es decir, antes y después del acuerdo de operaciones conjuntas entre Varig y Tam. Las opciones no son solamente las empresas que actúan en esta ruta, sino también se deben considerar los aeropuertos de origen y destino, además de la eventual existencia de escalas.

En octubre de 2004, en la ruta Santos DumontCongonhas (SDU-CGH), volando sin escalas, existían las conexiones por Varig, Tam, Vasp y Gol. En esta misma ruta, en abril de 2003, operaban Varig, Tam, Vasp y Gol. También operaba Ocean Air con aviones turbohélice; sin embargo, los vuelos hacían una o dos escalas (Guarulhos y San José dos Campos, localizados en el estado de Sao Paulo). Cabe destacar que el objetivo de esa compañía no es la ruta Río de Janeiro-Sao Paulo, sino la ciudad de Sao Paulo y el polo petrolífero de Macaé (Estado de Río de Janeiro) con escala en la ciudad de Río de Janeiro.

En octubre de 2002, en la ruta Santos Dumont-Guarulhos (SDU-GRU) operaba apenas Ocean Air sin escalas. En abril de 2003, la misma compañía continuaba en operación en la ruta sin escalas, pero con la introducción de un vuelo con escalas.

En los dos momentos considerados, en la ruta GaleónCongonhas (GIG-CGH) existía la opción entre Tam y Gol. El cambio principal entre estos dos momentos se refiere al reemplazo de aeronaves efectuada por la Tam, que cambió los Fokker F-100 por los Airbus A320.

Finalmente, en la ruta Galeón-Guarulhos (GIG-GRU) se mantuvieron las opciones Varig, Tam, Vasp y Fly. Se debe destacar que, en esta ruta, el acuerdo entre Tam y Varig no estaban en vigor las fechas en estudio, ya que los vuelos de Varig no tienen como objetivo principal la explotación de la ruta. La compañía realiza esta conexión apenas como una extensión de vuelos internacionales, en la mayor parte de las veces con aviones de gran envergadura (MD11 y Boeing 767). Por otro lado, la compañía Fly, que operaba vuelos de bajo costo con Boeing 727, dejó de tener frecuencia diaria en esta ruta en 2003 (y posteriormente cerró sus operaciones), lo que justifica el uso de un número fraccional para representar la cantidad de vuelos diarios. Este número es la media de vuelos en días útiles. 
Debido al acuerdo de cooperación entre Varig y Tam, en abril de 2003, las dos compañías en la ruta SDU-CGH forman una única DMU. A pesar de que, en octubre de 2002, todavía no existía el acuerdo de cooperación para efectos de comparación, se creó una unidad ficticia que considera la agregación de los datos de las dos compañías en la ruta citada.

Se consideraron dos envolventes o fronteras: la frontera tradicional (u optimista) y la invertida (o pesimista). La primera frontera también se puede interpretar como una óptica del pasajero, pues éste busca obtener el máximo de beneficios con el menor costo posible. Por otro lado, la frontera invertida, de cierta forma, puede ser vista como la óptica del empresario, pues considera eficiente a la DMU que obtiene el máximo de lucro con los recursos ofrecidos. Así, mientras el precio del pasaje es un input en la primera óptica (que debe ser minimizado), se convierte en un output en la óptica del empresario, con el objetivo de maximización de su valor.

En la óptica del pasajero, la variable precio es considerada input. Cuando una unidad tenía precios diferentes en función de los horarios de los vuelos, se utilizó el precio promedio. Como outputse debe observar lo que el pasajero pretende obtener con el costo del pasaje. En [15] se realizó un análisis exhaustivo de los beneficios que el pasajero espera por el valor pagado por el pasaje; sin embargo, en este artículo se consideran apenas los dos aspectos más relevantes. Como el pasajero de esa ruta tiene perfil predominantemente de ejecutivo, se considera que su preferencia es por la flexibilidad de horario (medida por el número de vuelos diarios) y por la adecuación de la ruta a sus objetivos [15]. Este output es subjetivo y sólo puede ser incluido en el modelo después de ser cuantificado.

Por otro lado, el empresario se preocupa en obtener el máximo de lucro ofreciendo lo mínimo posible. Así, en este caso, los inputs son número de vuelos y rutas, y el output es el precio practicado.

Otros factores, como la aeronave utilizada, el servicio a bordo y el programa de millas son menos relevantes. Además, estos factores son de difícil análisis, en virtud del propio acuerdo Tam-Varig, pues un pasajero puede adquirir el pasaje en una de las compañías, ganar millas en el programa de esa compañía y volar por aviones y con servicio a bordo de la otra. Por lo tanto, esos aspectos no fueron considerados en este estudio.

La adecuación de la ruta se evaluó usando el método MACBETH [3], el cual permite construir una escala cardinal de valores o función de valor monoatributo basado en opiniones subjetivas. Una revisión completa de este método puede ser encontrada en [16].

Los outputs cuantificados con el uso del método MACBETH fueron las siguientes opciones de conexiones aérea: SDU-CGH sin escalas (SDU-CGH-s), SDU-CGH con una escala (SDU-CGH-1), SDU-CGH con dos escalas (SDU-CGH-2), SDU-GRU sin escalas (SDU-GRU-s), SDU-GRU con una escala (SDU-GRU-1), GIG-GRU sin escalas (GIG-GRU-s) y GIG-CGH sin escalas (GIG-CGH-s).

En la figura 1 se muestra el gráfico de la relación de preferencia entre las opciones de ruta, en el cual las flechas representan el sentido de la preferencia; en el caso de doble flecha, las alternativas son consideradas indiferentes. En este gráfico, como el decidor siguió el principio de racionalidad fuerte [5], existe transitividad de preferencias (si $A$ es preferible a $B$ y $B$ es preferible a $C$, entonces $A$ es preferible a $C$ ), tal como se definió en [10]. De esta forma, se eliminaron las aristas redundantes del gráfico para minimizar el efecto da contaminación visual de la figura.

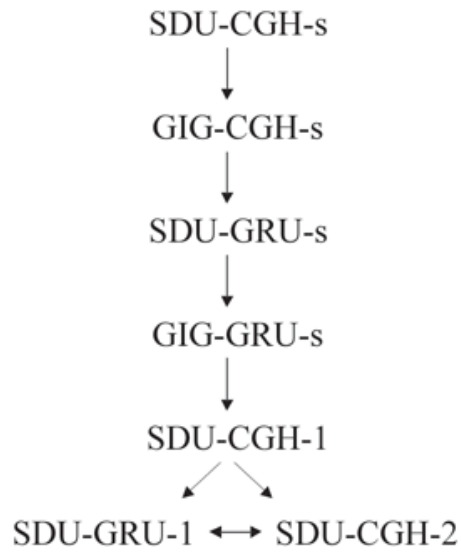

Fig. 1 Gráfico de la relación de preferencia entre las opciones de ruta.

La tabla 1 muestra las unidades, DMUs, que serán evaluadas y los valores de las variables (inputs y outputs) para los dos casos. La columna "Rutas" muestra los valores cuantificados en una escala cardinal después de emitir los juicios de valor y de aplicar el método MACBETH ya mencionado. Para obtener esta escala, además de la relación de preferencia mostrada en la figura 1 , se consideró que, para el pasajero promedio, es menos perjudicial cambiar SDU por GIG que cambiar CGH por GRU. 
En este artículo se utilizó el modelo DEA CCR, pues no existen evidencias de ganancias o pérdidas de escala, según la opinión del pasajero. Los outputs, aun después de haber sido cuantificados, miden la calidad del servicio prestado y modelos DEA con variables de calidad pierden sentido con el empleo de retornos variables de escala.

Cabe destacar que la posición del empresario sirve apenas para calcular la ineficiencia y mejorar la ordenación, con el uso del modelo de dos fronteras y la agregación de la ineficiencia con relación a la frontera invertida. El resultado final mide la eficiencia de los servicios de cada DMU para el pasajero.

\section{Resultados}

La tabla 2 contiene los resultados del modelo DEA utilizado. La columna "Eficiencia ${ }_{\text {pasajero }}$ "mide la eficiencia según la óptica del pasajero, es decir, con relación a la envolvente superior. La tercera columna,

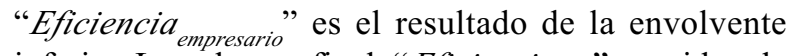
inferior. La columna final, "Eficiencia final " considera la agregación del índice de la segunda columna y del complemento de la tercera. Los cálculos de esa tabla fueron realizados con el uso del software SIAD [1].

Se puede observar que la operación de Varig y Tam anterior al acuerdo de cooperación es mucho más atractiva para el pasajero que la misma operación después del acuerdo. Las DMUs correspondientes tienen como índices finales 76,8 y $65,4 \%$, respectivamente.

Es importante resaltar que no fueron consideradas las diferencias de coyuntura macroeconómica entre los dos momentos. Sin embargo, se verifica que las dos opciones de conexión aérea Rio de Janeiro-Sao Paulo más atrayentes son Vasp y Gol en la ruta SDU-CGH, ambas en abril de 2003. Esto muestra que la situación macroeconómica no impide la mejora entre octubre de 2002 y abril de 2003.

Tabla 1 DMUs, inputs y outputs para el problema estudiado.

\begin{tabular}{|c|c|c|c|}
\hline DMU & Precios (R\$) & Número de vuelos & Rutas \\
\hline VARIG-TAM SDU-CGH 2003 & 306,20 & 44 & 100,0 \\
\hline TAM GIG-CGH 2003 & 192,25 & 3 & 83,3 \\
\hline TAM GIG-GRU 2003 & 218,54 & 7 & 47,9 \\
\hline VASP SDU-CGH 2003 & 163,00 & 9 & 100,0 \\
\hline VASP GIG-GRU 2003 & 163,00 & 2 & 47,9 \\
\hline GOL SDU-CGH 2003 & 168,60 & 10 & 100,0 \\
\hline GOL GIG-CGH 2003 & 178,25 & 4 & 83,3 \\
\hline VARIG GIG-GRU 2003 & 192,25 & 4 & 47,9 \\
\hline FLY GIG-GRU 2003 & 110,00 & 1 & 47,9 \\
\hline OCEAN AIR SDU-GRU 2003, sin escalas & 156,20 & 2 & 66,7 \\
\hline OCEAN AIR SDU-GRU 2003, con 1 escala & 156,20 & 1 & 25,0 \\
\hline OCEAN AIR SDU-CGH 2003, con 1 escala & 156,20 & 1 & 25,5 \\
\hline OCEAN AIR SDU-CGH 2003, con 2 escala & 156,20 & 1 & 100,0 \\
\hline VARIG SDU-CGH 2002 & 287,00 & 36 & 47,9 \\
\hline VARIG GIG-GRU 2002 & 211,25 & 8 & 83,3 \\
\hline TAM GIG-CGH 2002 & 211,25 & 4 & 47,9 \\
\hline TAM GIG-GRU 2002 & 211,25 & 4 & 100,0 \\
\hline TAM SDU-CGH 2002 & 294,20 & 26 & 47,9 \\
\hline VASP GIG-GRU 2002 & 237,00 & 4 & 100,0 \\
\hline VASP SDU-CGH 2002 & 237,00 & 16 & 83,3 \\
\hline GOL GIG-CGH 2002 & 159,00 & 4 & 100,0 \\
\hline GOL SDU-CGH 2002 & 219,00 & 12 & 47,9 \\
\hline FLY GIG-GRU 2002 & 65,40 & 0,6 & 66,7 \\
\hline OCEAN AIR SDU-GRU 2002, sin escalas & 228,00 & 1 & 100,0 \\
\hline VARIG-TAM SDU-CGH 2002, ficticia & 290,02 & 62 & \\
\hline
\end{tabular}


Tabla 2 Resultados del modelo propuesto (eficiencia en porcentajes).

\begin{tabular}{|c|c|c|c|}
\hline DMU & Eficiencia $_{\text {pasajero }}$ & Eficiencia $_{\text {empresário }}$ & Eficiencia $_{\text {final }}$ \\
\hline VASP SDU-CGH 2003 & 95,8 & 26,1 & 84,8 \\
\hline GOL SDU-CGH 2003 & 94,1 & 27,0 & 83,6 \\
\hline VARIG-TAM SDU-CGH 2002, ficticia & 100,0 & 46,4 & 76,8 \\
\hline FLY GIG-GRU 2002 & 100,0 & 47,8 & 76,1 \\
\hline GOL GIG-CGH 2002 & 76,2 & 30,6 & 72,8 \\
\hline GOL SDU-CGH 2002 & 74,7 & 35,1 & 69,8 \\
\hline VASP SDU-CGH 2002 & 73,3 & 37,9 & 67,7 \\
\hline GOL GIG-CGH 2003 & 68,0 & 34,3 & 66,9 \\
\hline VARIG SDU-CGH 2002 & 78,2 & 45,9 & 66,1 \\
\hline VARIG-TAM SDU-CGH 2003 & 79,9 & 49,0 & 65,4 \\
\hline TAM GIG-CGH 2003 & 61,7 & 39,8 & 61,0 \\
\hline TAM SDU-CGH 2002 & 67,7 & 47,1 & 60,3 \\
\hline TAM GIG-CGH 2002 & 57,4 & 40,6 & 58,4 \\
\hline OCEAN AIR SDU-GRU 2003, sin escalas & 60,2 & 45,8 & 57,2 \\
\hline FLY GIG-GRU 2003 & 60,4 & 56,2 & 52,1 \\
\hline VASP GIG-GRU 2003 & 42,3 & 54,5 & 43,9 \\
\hline VARIG GIG-GRU 2003 & 38,5 & 64,2 & 37,1 \\
\hline VARIG GIG-GRU 2002 & 39,8 & 70,6 & 34,6 \\
\hline TAM GIG-GRU 2002 & 35,0 & 70,6 & 32,2 \\
\hline TAM GIG-GRU 2003 & 37,3 & 73,0 & 32,2 \\
\hline VASP GIG-GRU 2002 & 31,2 & 79,2 & 26,0 \\
\hline OCEAN AIR SDU-CGH 2003, con 1 escala & 33,6 & 87,9 & 22,9 \\
\hline OCEAN AIR SDU-GRU 2002, sin escalas & 40,1 & 100,0 & 20,1 \\
\hline OCEAN AIR SDU-CGH 2003, con 2 escala & 23,0 & 100,0 & 11,5 \\
\hline OCEAN AIR SDU-GRU 2003, con 1 escala & 23,0 & 100,0 & 11,5 \\
\hline
\end{tabular}

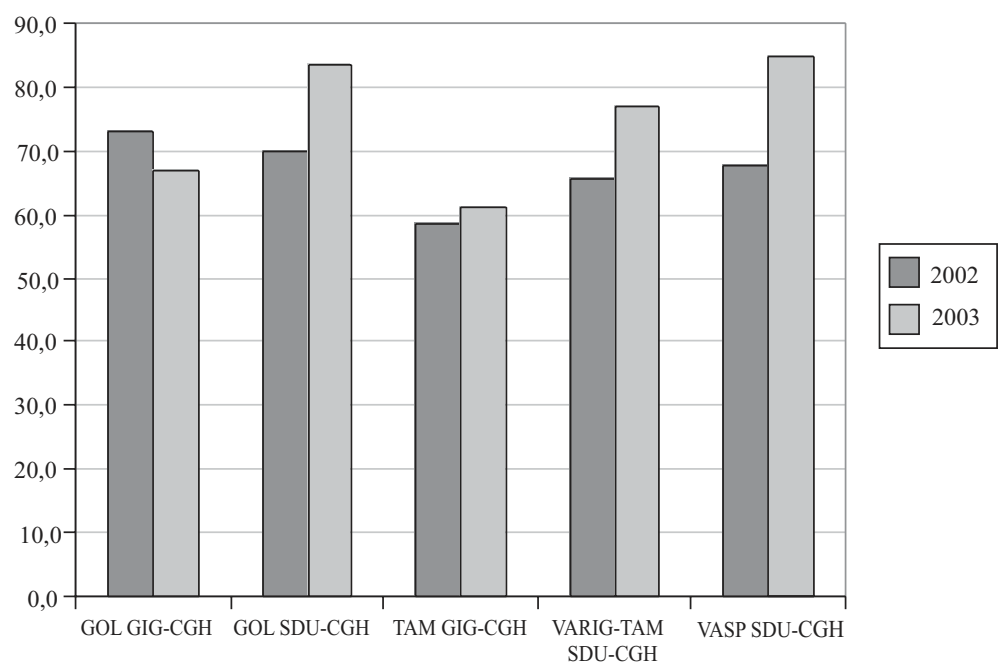

Fig. 2 Comparación de algunas opciones de conexión aérea Río de Janeiro-Sao Paulo, en 2002 y en 2003. 
La figura 2 compara algunas de las opciones principales en los dos momentos evaluados. Con excepción de las dos compañías que realizaron el acuerdo de cooperación, se nota un aumento de eficiencia en 2003 en la ruta SDU$\mathrm{CGH}$ y una casi indiferencia en la ruta GIG-CGH.

\section{CONCLUSIONES}

El modelo DEA de doble envolvente utilizado en este artículo se mostró útil para mejorar la discriminación entre DMUs eficientes. Este modelo impide que una DMU sea evaluada apenas por sus resultados más favorables y también obliga a tomar en cuenta los resultados menos favorables.

Los resultados analizados muestran que, por lo menos en un primer momento, el acuerdo entre Varig y Tam es perjudicial para los pasajeros. No existen evidencias de que los resultados obtenidos hayan sido influenciados por los cambios en la coyuntura macroeconómica.

Finalmente, cabe destacar que la frontera inferior se determina por las opciones de vuelos con escala, lo que provoca una gran distancia entre las dos fronteras envolventes. La retirada de esas opciones podría acarrear cambios en el índice de eficiencia final. Ésta es una característica de los modelos que son altamente sensibles a los cambios en el conjunto de DMUs observadas.

Es importante destacar que el estudio mostrado en este artículo es válido apenas para los momentos en que fue efectuado. Sucedieron evoluciones posteriores, como la salida de la empresa Fly, el fin de la ruta GRU-SDU por Ocean Air, la disminución de vuelos de VASP (actualmente suspendido temporalmente), el fin de la conexión GIG-CGH por Tam, el reordenamiento de vuelos entre SDU y GIG, entre otros. Aunque no fue realizado ningún estudio cuantitativo sobre estos cambios, un primer análisis cualitativo indica que las opciones del pasajero fueron reducidas, lo que concuerda con los resultados obtenidos en este artículo para los primeros momentos de operación conjunta de Varig y TAM.

\section{AGRADECIMIENTOS}

Al CNPq (procesos 471014/2003-6 y 306924/2004-8) por el apoyo financiero.

\section{REFERENCIAS}

[1] L. Angulo-Meza, L. Biondi Neto, J.C.C.B. Soares de Mello, E.G. Gomes, P.H.G. Coelho. "FSDAFree Software for Decision Analysis (SLADSoftware Livre de Apoio a Decis,,o): A Software Package for Data Envelopment Analysis Models". Congreso Latino-Iberoamericano de Investigación de Operaciones y Sistemas. La Habana, Cuba. 2004.

[2] L. Angulo-Meza, M.P. Estellita Lins. "Review of Methods for Increasing Discrimination in Data Envelopment Analysis". Annals of Operations Research. Vol. 116, pp. 225-242. 2002.

[3] C.A. Bana e Costa, J.C. Vansnick. "A theoretical framework for Measuring Attractiveness by a Categorical Based Evaluation Technique (MACBETH)". In: Clímaco, J. (Ed.) Multicriteria Analysis. Berlin: Springer Verlag: 1995.

[4] R.D. Banker, A. Charnes, W.W. Cooper. "Some models for estimating technical scale inefficiencies in Data Envelopment Analysis". Management Science. Vol. 30. N ${ }^{\circ}$ 9, pp. 10781092. 1984.

[5] A. Barba-Romero, J.C. Pomerol. "Decisiones Multicriterio: Fundamentos Teóricos y Utilización Práctica”. España: Colección de Economía, Universidad de Alcalá. 1997.

[6] A. Charnes, W.W. Cooper, E. Rhodes. "Measuring the efficiency of decision-making units". European Journal of Operational Research. Vol. 2, pp. 429-444. 1978.

[7] R. Coelho. "A construção da agenda de desregulamentação do setor de transporte aéreo no Brasil". Panorama Nacional de Pesquisa em Transportes. Vol. 2, pp. 383-394. 2002.

[8] T. Entani, Y. Maeda, H. Tanaka. "Dual Models of Interval DEA and its extensions to interval data". European Journal of Operational Research. Vol. 136, pp. 32-45. 2002.

[9] R.A. Espírito Santo Jr. "Concentração no transporte aéreo e os possíveis impactos sobre os consumidores, a sociedade e a economia". Transporte em Transformação VI, pp. 155-171. 2002. 
[10] L.F.A.M. Gomes, M.C. Gonzalez-Araya, C. Carignano. "Tomada de Decisões em Cenários Complexos". Editorial Thomsom. 2003.

[11] F.R. Leta, J.C.C.B. Soares de Mello, E.G. Gomes, L. Angulo-Meza. "Métodos de Melhora de Ordenação em DEA Aplicados à Avaliaç,,o Estática de Tornos Mecânicos”. Investigaç,,o Operacional. Vol. 25. 2005.

[12] M.P.E. Lins, L.F.L. Novaes, L.F.L. Legey. "Real estate appraisal: a double perspective data envelopment analysis approach". Annals of Operations Research, Vol. 138, pp. 79-96. 2005.

[13] A. Oliveira, F. Turolla, A. Busto. "Modelagem dos Impactos da Política de Flexibilização na Competição das Companhias Aéreas Brasileiras". In: XXX Congresso ANPEC, Nova Friburgo. Anais. Río de Janeiro. 2002.
[14] S. Oliver, "Novos Rumos na aviação comercial brasileira". Avião Revue. No 42, pp. 22-27. 2003.

[15] J.C.C.B. Soares de Mello, E.G. Gomes, L.F.A. Gomes, L. Biondi Neto, F.R. LETA. "Seleção de ruta aérea con o uso do Apoio Multicritério à Decisão". Engevista. Vol. 5. No 10, pp. 71-84. 2003.

[16] J.C.C.B. Soares de Mello, E.G. Gomes, M.P. Estellita Lins. "Análise Multicritério da presença da Universidade Federal Fluminense con o uso do Método MACBETH". Revista Produção. Vol. 11. No 2, pp. 53-67. 2002.

[17] Y. Yamada, T. Matui, M. Sugiyama. "New analysis of efficiency based on DEA". Journal of the Operations Research Society of Japan. Vol. 37. No2, pp. 158-167. 1994. 